\title{
Bfomédica
}

Articulo Original

\section{Modelado espacial bayesiano de la enfermedad de Chagas en la Huasteca Potosina}

Hugo Medina-Garza, Carlos Contreras-Servín, María G. Galindo-Mendoza, José de Jesús Mejía-Saavedra, Beatriz E. Arreola-Martínez

Universidad Autónoma de San Luis Potosí

\section{Autor para correspondencia}

Nombre: Beatriz Estrella Arreola Martínez

Institución: Universidad Autónoma de San Luis Potosí

Dirección: Av. Sierra Leona No. 550 Col. Lomas Segunda sección C.P. 78220, San Luis Potosí, San Luis Potosí, México

E-mail: beatriz.arreola@uaslp.mx

Recibido: el 22 de febrero de 2016 Aceptado para publicación: el 20 de mayo de 2016

Copyright (C) 2016 por autores (s) y Revista Biomédica.

Este trabajo esta licenciado bajo las atribuciones de la Creative Commons (CC BY).

http://creativecommons.org/licenses/by/4.0/ 


\section{Resumen}

Introducción. La enfermedad de Chagas es una infección sistémica, causada por el protozoario Trypanosoma cruzi. Está documentado como la influencia de factores ambientales favorecen la presencia de la infección.

Objetivo. Comprender los patrones de distribución espacial de riesgo y los factores ambientales y socioeconómicos que favorecen la propagación de la enfermedad de Chagas.

Material y métodos. Se aplicó un análisis de regresión espacial bayesiano a datos de la incidencia de la enfermedad de Chagas en la Huasteca Potosina en el periodo de 2003-2012.

Resultados. Se observó un patrón claro de distribución espacial en la incidencia de la enfermedad en la Huasteca Potosina, misma que se vio significativamente correlacionada en el modelo de regresión multivariante final con la temperatura mínima, los porcentajes de viviendas con piso de tierra, de viviendas sin agua entubada, de población de tres años y más que habla lengua indígena y no habla español, de población mayor a 15 años analfabeta, derechohabiente a servicios de salud, de viviendas con algún nivel de hacinamiento y de viviendas habitadas que cuentan con servicios.

Conclusiones. El análisis pone de manifiesto que es la población indígena la más afectada, ya que este grupo es el que más carece de acceso a servicios (salud, educación, vivienda), por lo tanto, favorece la presencia del vector causante de la enfermedad. En la Huasteca Potosina, esta población habita en zonas de difícil acceso, lo que dificulta la implementación de programas adecuados de control del vector y la infección.

Palabras Clave: enfermedad de Chagas, Huasteca Potosina, modelado bayesiano

\section{Abstract}

Bayesian spatial modeling of Chagas disease in the Huasteca Potosina Introduction. Chagas disease is a systemic infection caused by the protozoan Trypanosoma cruzi. There is evidence that environmental factors favor the maintenance of the infection.

Objective. To understand the spatial distribution patterns of risk, and environmental and socioeconomic factors that favour the spread of Chagas disease.

Material and methods. Data on Chagas disease incidence in the Huasteca Potosina were collected during 2003-2012 and then analyzed by a Bayesian method of spatial regression. 
Results. A clear pattern of spatial distribution of incidence of the disease in the Huasteca was observed, and it was significantly correlated with the final multivariate regression model using variables such as the minimum temperature, $\%$ of households with dirt floors, $\%$ of households without running water, $\%$ of population with three years and over speaking indigenous languages only, \% of population over 15 years illiterate, \% entitled to health care, $\%$ of households with some level of overcrowding and \% of households with basic services.

Conclusions. The analysis showed that the indigenous population is the most affected by Chagas disease and that was related to lack to basic services (health, education, housing), therefore, those conditions favor not only the presence of the vector but also the disease. In the Huasteca, this population lives in areas of difficult access, which hinders the implementation of appropriate programs to control the vector and the infection.

Keywords: Chagas disease, Huasteca Potosina, Bayesian modeling

\section{INTRODUCCIÓN}

La enfermedad de Chagas o Tripanosomiasis americana es una enfermedad transmitida por vectores, principalmente triatominos de la familia Reduviidae, orden Hemiptera, Subfamilia Triatominae. Es una infección endémica causada por el parásito protozoario Trypanosoma cruzi (T. cruzi). Esta enfermedad representa el principal problema de salud pública en América Latina. Según estimaciones recientes, en todo el mundo hay alrededor de ocho millones de personas infectadas (1).

Esta enfermedad se encuentra asociada a la pobreza y las malas condiciones de la vivienda; se localiza ampliamente distribuida, en las áreas rurales de Latinoamérica y en zonas marginadas de las grandes ciudades principalmente (2). La enfermedad de Chagas, más que ninguna otra, está íntimamente ligada con el desarrollo económico y social (3). A partir de 1993 la OMS la consideró como la enfermedad parasitaria más grave en América y es parte de la lista de las catorce enfermedades "descuidadas o desatendidas".

La infección con T. cruzi puede ser transmitida a los humanos de manera congénita y connatal, por transfusiones de sangre o trasplantes de órganos y por vía oral (4,5); sin embargo, el principal mecanismo de transmisión es vectorial (6). Los triatominos (también conocidos como chinche, chinche besucona, pic, talaje u otros nombres dependiendo de la región geográfica) son los vectores 
naturales de T. cruzi, que pasa al huésped cuando la chinche se alimenta de sangre y excreta heces infectadas con el parásito, permitiendo que el protozoo penetre por las heridas o mucosas (7). Su importancia epidemiológica se basa en la elevada adaptabilidad del vector, debido a que habita diversos ecosistemas, entre las que se incluyen selvas húmedas y secas (8), diferentes altitudes (50$1580 \mathrm{msnm}$ ) (9), explota diversas fuentes alimenticias (10), tiene alta diversidad en sitios de descanso (11-13) gran capacidad de colonización de las viviendas $(14,15)$ y alta longevidad, puede pasar varios días sin alimento (510 días en condiciones de laboratorio) y capacidad de dispersión (16,17). Estos factores tienen un efecto directo sobre las tasas de transmisión de T. cruzi a humanos.

Además, los factores socioeconómicos pueden influir en la transmisión: una mayor incidencia tiende a ocurrir en zonas rurales, con población indígena y marginada. En San Luis Potosí, de acuerdo a los datos de la Secretaría de Salud del Estado, se han reportado un total de 257 infectados en el periodo de 2003 al 2012, siendo la Jurisdicción Sanitaria Número V y VI (correspondiente a la región Huasteca) registran el mayor número de casos (de acuerdo a datos proporcionados por la Secretaría de Salud del Estado de San Luis Potosí vía INFOMEX).

El aumento de la frontera humana también puede incrementar la incidencia de la enfermedad, al entrar en contacto directo con los insectos vectores (18). Por lo tanto, podemos suponer que la distribución espacial de la tripanosomiasis americana se determina por factores ambientales (incluido el uso de suelo y cambios en el uso de suelo), que influyen en la distribución de las chinches, y factores socioeconómicos, que pueden alterar el patrón de contacto entre los humanos y los vectores.

Pocos estudios han analizado la distribución espacial de la tripanosomiasis americana con relación a las condiciones ambientales y características socioeconómicas de la población, a pesar de la importancia de estos factores en la transmisión de enfermedades. Dumonteil y Gourbiere (19) exploraron las relaciones entre la distribución geográfica de Triatoma dimidiata y factores bioclimáticos en la península de Yucatán, México para obtener el primer mapa de riesgo de transmisión natural de la enfermedad de Chagas en esa zona, pero no incluyeron variables socioeconómicas de la población en su análisis.

A continuación se presenta un análisis espacial de la incidencia de tripanosomiasis americana en San Luis Potosí entre 2003 y 2012 que considera variables climáticas y socioeconómicas. Se incorporó un marco bayesiano, con inclusión de efectos aleatorios correlacionados y no correlacionados espacialmente para estimar rigurosamente la variación espacial en el riesgo de la enfermedad, mientras se cuantificaron los efectos de las covariables. Este tipo de enfoque se utiliza comúnmente para estimar la variación espacial en el riesgo de enfermedad en áreas pequeñas (por 
ejemplo, áreas administrativas) y garantiza la plena representación de la incertidumbre en los parámetros estimados $(20,21)$. Por lo tanto, esta investigación, explora la influencia de variables ambientales y socioeconómicas en la distribución espacial de la enfermedad de Chagas en la Huasteca Potosina, y proporcionará una mayor comprensión del patrón de riesgo de esta enfermedad.

\section{MATERIALES Y METODOS}

La investigación se centró en la Huasteca Potosina, localizada en la parte oriental del Estado de San Luis Potosí, México. Tiene como límites por el occidente la sierra madre oriental y los estados de Tamaulipas al norte, Veracruz al este e Hidalgo y Querétaro por el sur. El área se encuentra delimitada en las coordenadas geográficas $22^{\circ} 40^{\prime}$ latitud norte, $99^{\circ} 40^{\prime}$ longitud oeste en el municipio de Ciudad del Maíz; $22^{\circ} 25^{\prime}$ latitud norte, $98^{\circ} 40^{\prime}$ longitud oeste en el municipio de Ébano; $21^{\circ} 34^{\prime}$ latitud norte, $99^{\circ} 43^{\prime}$ longitud oeste en el municipio de San Ciro Acosta y $21^{\circ} 08^{\prime}$ latitud norte, $98^{\circ} 50^{\prime}$ longitud oeste en el municipio de Tamazunchale. En esta región predominan climas cálidos húmedos y subhúmedos y semicálidos húmedos. Se caracteriza por tener población indígena y presentar un grado de marginación medio y alto (el $61 \%$ de la población presenta grado de marginación alto y muy alto), además de que las condiciones de las viviendas de estas zonas (22) presentan las características idóneas para albergar a los vectores de la enfermedad de Chagas. (Figura 1).

Los datos sobre el número de enfermos de Chagas y municipios donde se presentaron los casos entre 2003 y 2012 se obtuvieron mediante una solicitud de información a través de INFOMEX. La base de datos proporcionada contenía información por año y por municipio del total de casos registrados de cada una de las localidades de la Huasteca Potosina que presentaron casos de enfermos de Chagas en el periodo 2003-2012. Se utilizó además una base de datos de variables ambientales (temperatura mínima, máxima, media, precipitación) y socioeconómicas (población total, índice de marginación, porcentaje de viviendas con piso de tierra, porcentaje de viviendas sin agua entubada, sin drenaje, sin energía eléctrica, población de tres años y más que habla lengua indígena y no habla español, población de 15 años y más analfabeta, población derechohabiente y no derechohabiente a servicios de salud, población con hogares censales indígenas, población ocupada con ingresos de hasta dos salarios mínimos, población ocupada, \% de viviendas con algún nivel de hacinamiento, viviendas particulares habitadas con luz, agua y drenaje, viviendas particulares habitadas sin ningún bien). El conjunto de datos climáticos fue obtenido de la red de estaciones meteorológicas de la Comisión Nacional del Agua (CNA). Se usaron las normales climatológicas de 1981-2010 para obtener la temperatura mínima, media y máxima (en grados centígrados, ${ }^{\circ} \mathrm{C}$ ) y la precipitación media anual (en milímetros, $\mathrm{mm}$ ). Se extrajeron los valores medios de temperatura y precipitaciones de toda la zona usando ArcMap 9.3 (ESRI) (23). La información socioeconómica fue obtenida del censo de 
población y vivienda 2010 del Instituto Nacional de Estadística y Geografía (INEGI) a excepción del índice de marginación y las variables usadas para su cálculo, que se obtuvieron del Consejo Nacional de Población (CONAPO).

La correlación entre la enfermedad de Chagas y cada una de las covariables enumeradas anteriormente se examinó usando diagramas de dispersión y la regresión Poisson univariado en el paquete estadístico R. Las variables que fueron significativamente correlacionadas con el resultado fueron evaluadas para la co-linealidad con otras variables significativas. Cuando dos o más variables que fueron medidas de fenómenos relacionados (por ejemplo temperatura mínima y temperatura promedio) producían una correlación alta $(>0.75)$, la variable con el más pequeño valor del criterio de Akaike (AIC) en el modelo de regresión univariada fue seleccionada para usarse y las otras fueron omitidas. Cuando dos o más variables estaban fuertemente correlacionadas, pero eran consideradas mediciones de fenómenos no relacionados, se utilizaron en la comparación de modelos en el análisis de regresión de Poisson no espacial.

Una regresión de Poisson multivariada se llevó a cabo utilizando las covariables que se encontraron asociados significativamente durante el análisis exploratorio $(\mathrm{p} \leq 0.2$ de la regresión de Poisson univariada). Los modelos candidatos contenían una de las covariables marcadas como colineales entre sí (Cuadro 1), junto con todas las otras covariables, no colineales (Cuadro 1). Cualquier variable que no fue estadísticamente significativa ( $p>0.05)$ se eliminó posteriormente del modelo; si múltiples variables no fueron significativas, la variable con el mayor valor de $\mathrm{p}$ fue eliminada primero, se evaluó la significancia del resto de las variables y después, si era necesario, se eliminaron las variables adicionales. Para comprobar la autocorrelación espacial de los residuos del modelo de Poisson multivariado (lo que indicaría que las covariables en el modelo no capturaron completamente el patrón espacial de la enfermedad de Chagas) se calculó el estadístico I de Moran usando los residuales de Poisson.

Las influencias de autocorrelación espacial (donde las observaciones más cercanas en el espacio son más propensas a tener valores similares de observaciones que están muy separados) se abordaron, y la sobredispersión en los datos fue contabilizada, utilizando un modelo de regresión de Poisson lognormal con un efecto aleatorio no espacial y un efecto aleatorio estructurado espacialmente dependiente utilizando un modelo condicional autoregresivo previo (CAR) $(21,24)$. El componente CAR utilizó una matriz de adyacencia espacial sencilla para representar relaciones espaciales entre áreas administrativas. Efectos aleatorios no espaciales también se incluyeron en la comparación de 
modelos. Las covariables seleccionadas en el análisis de regresión de Poisson multivariante no espacial descrito anteriormente se incluyeron como efectos fijos en esta etapa del análisis.

El modelo, realizado en WinBugs, asumió que la cuenta observada de casos de enfermos de Chagas yi por municipio i ( $=1$ a 20) seguido de la distribución Poisson con una media de $\mu \mathrm{i}$ :

$$
\begin{aligned}
& \text { yi } \sim \text { Poisson }(\mu \mathrm{i}) \\
& y \\
& \log \mu \mathrm{i}=\log \mathrm{Ei}+\theta \mathrm{i}
\end{aligned}
$$

donde Ei (el número de casos esperados de la enfermedad de Chagas en el área administrativa i) es un término de desplazamiento utilizado para el control del tamaño de la población dentro de las áreas administrativas y $\theta \mathrm{i}$ es el log relativo de riesgo.

El logaritmo relativo del riesgo fue modelado así:

$$
\theta \mathrm{i}=\alpha+\beta 1 \ldots \mathrm{iC} 1 \ldots \mathrm{i}+\mathrm{Si}+\mathrm{Ui}
$$

donde $\alpha$ es el término de intersección; $\beta 1$...i es un vector de coeficientes de las covariables, $\mathrm{C} 1 \ldots \mathrm{i}$; Si un efecto aleatorio espacialmente correlacionados estructurado utilizando CAR previo de que se le asignó una sencilla matriz de adyacencias espacial (para identificar qué polígonos son directamente adyacentes entre sí) y precisión $\tau$; y Ui un efecto aleatorio no correlacionada espacialmente con media cero y precisión $\tau$.

Después de un periodo de ablande inicial de 10.000 iteraciones, se realizaron otras 200.000 iteraciones, y cada décima iteración se almacenó durante la estimación de parámetros para reducir la autocorrelación en las muestras. Las cadenas de Markov-Monte Carlo (MCMC) fueron inspeccionados para comprobar la convergencia y múltiples valores iniciales para cada parámetro se utiliza para asegurar que los algoritmos MCMC convergían en el mismo espacio de parámetros desde diferentes puntos de partida. Medidas de resumen para la distribución posterior de cada parámetro (posterior media, desviación estándar y el 95\% intervalo de credibilidad [CrI]) se almacenaron para proporcionar estimaciones de los parámetros.

Se compararon cuatro modelos candidatos: (i) solo los efectos fijos; (ii) los efectos fijos y efectos aleatorios no espaciales; (iii) los efectos fijos y efectos aleatorios espacialmente correlacionados; y (iv) los efectos fijos, efectos aleatorios espacialmente correlacionadas y efectos aleatorios no espaciales. Los modelos se compararon mediante el criterio de desviación de la información (DIC): 
el modelo con el criterio más bajo DIC fue seleccionado como el de mejor ajuste. Todas las covariables, incluyendo aquellos que no mantienen la significancia estadística $(p>0,05)$, fueron retenidos para proporcionar un resumen de todos los efectos de la covarianza.

\section{RESULTADOS}

De 2003 a 2012 se reportaron un total de 257 casos de enfermos de Chagas en la Huasteca Potosina con una tasa media de incidencia de 116.97 casos por año por cada 10,000 habitantes. Dentro de los 20 municipios de la Huasteca Potosina, los recuentos acumulativos de casos oscilaron entre 1 y 50 con un promedio de 12.85 y una mediana de 9.5. Una comparación las figuras 2 y 3 muestra una alta incidencia de la enfermedad en áreas donde el porcentaje de hogares donde el jefe del hogar o su cónyuge hablan alguna lengua indígena (principalmente Náhuatl, Huasteco y Pame). (Figuras 2 y 3 )

A excepción de porcentaje de viviendas sin drenaje, porcentaje de viviendas sin energía eléctrica, población con 2 salarios mínimos y viviendas sin bienes $(p>0.05)$, el resto de las variables se correlacionaron significativamente con la enfermedad de Chagas usando una regresión no espacial de Poisson $(\mathrm{p}<0.05)$ (Cuadro 1).

Cuadro 1. Parámetros del análisis de regresión Poisson univariado

\begin{tabular}{|c|c|c|c|c|c|}
\hline Variable & Estimado & Intervalo de confianza & Valor $\mathrm{z}$ & $\mathrm{p}$ & $\mathrm{AIC}$ \\
\hline Índice de marginación & 0.23047 & $0.0418-0.4204$ & 2.386 & $*$ & 278.33 \\
\hline Población total & $7.427 \mathrm{e}-06$ & $4.8 e-06-9.7 e-06$ & 5.951 & $* * *$ & 255.07 \\
\hline Precipitación & 0.0004628 & $0.00015-0.00076$ & 2.956 & $* *$ & 275.36 \\
\hline Temperatura Máxima & 0.17023 & $0.0451-0.3062$ & 2.560 & $*$ & 276.74 \\
\hline Temperatura Media & 0.15344 & $0.0831-0.2326$ & 4.039 & $* * *$ & 262.87 \\
\hline Temperatura Mínima & 0.24215 & $0.1406-0.3631$ & 4.276 & $* * *$ & 254.26 \\
\hline$\%$ viviendas con piso tierra & 0.019308 & $0.0064-0.0317$ & 2.996 & $* *$ & 275.49 \\
\hline$\%$ viviendas sin agua entubada & 0.016529 & $0.0098-0.0233$ & 4.783 & $* * *$ & 260.4 \\
\hline$\%$ viviendas sin drenaje & -0.06813 & $-0.1659-0.0257$ & -1.39 & & 282.08 \\
\hline$\%$ viviendas sin energía eléctrica & 0.005844 & $-0.0182-0.0290$ & 0.485 & & 283.84 \\
\hline $\begin{array}{l}\text { Población de } 3 \text { años y más que } \\
\text { habla lengua indígena y no español }\end{array}$ & 0.06545 & $0.0329-0.0971$ & 4.003 & $* * *$ & 268.91 \\
\hline $\begin{array}{l}\text { Población de } 3 \text { años y más que } \\
\text { habla alguna lengua indígena }\end{array}$ & 0.014367 & $0.0093-0.0195$ & 5.557 & $* * *$ & 251.25 \\
\hline $\begin{array}{l}\text { Población de } 15 \text { años y más } \\
\text { analfabeta }\end{array}$ & 0.04635 & $0.0209-0.0719$ & 3.557 & $* * *$ & 271.25 \\
\hline $\begin{array}{l}\text { Población derechohabiente a } \\
\text { servicios de salud }\end{array}$ & -0.04781 & $-0.0603--0.0351$ & -7.45 & $* * *$ & 230.74 \\
\hline $\begin{array}{l}\text { Población con hogares censales } \\
\text { indígenas }\end{array}$ & 0.013501 & $0.0089-0.0182$ & 5.706 & $* * *$ & 247.42 \\
\hline $\begin{array}{l}\text { Población ocupada con ingresos de } \\
\text { hasta } 2 \text { salarios mínimos }\end{array}$ & -0.005306 & $-0.0165-0.0062$ & -0.91 & & 283.25 \\
\hline Población ocupada & 0.13462 & $0.0558-0.2158$ & 3.302 & $* * *$ & 272.63 \\
\hline
\end{tabular}




\begin{tabular}{|c|c|c|c|c|c|}
\hline $\begin{array}{l}\text { Población sin derechohabiente a } \\
\text { servicios de salud }\end{array}$ & 0.046424 & $0.0336-0.0590$ & 7.179 & $* * *$ & 234.73 \\
\hline $\begin{array}{l}\% \text { de viviendas con algún nivel de } \\
\text { hacinamiento }\end{array}$ & 0.036331 & $0.0173-0.0551$ & 3.775 & $* * *$ & 270.08 \\
\hline $\begin{array}{l}\text { Viviendas particulares habitadas } \\
\text { que disponen de luz, agua y } \\
\text { drenaje }\end{array}$ & -0.010554 & $-0.0105--0.0036$ & -2.932 & $* *$ & 274.99 \\
\hline $\begin{array}{l}\text { Viviendas particulares habitadas } \\
\text { sin ningún bien }\end{array}$ & 0.01658 & $-0.0049-0.0377$ & 1.521 & & 281.79 \\
\hline
\end{tabular}

La variable temperatura media fue excluida por presentar colinealidad con otras variables relacionadas con el fenómeno, lo mismo para la población sin derechohabiencia a servicios de salud. La variable viviendas con luz, agua y drenaje correlacionó de manera negativa con el total de casos, lo que era de esperarse, ya que estas viviendas tienen menos espacios para ser ocupados por las chinches. Lo mismo pasó con la variable población derechohabiente a servicios de salud, también correlacionó de manera negativa con el total de casos. El resto de las variables que correlacionaron de manera negativa no fueron significativas.

Cuadro 2. Modelo de regresión de Poisson multivariada final con algunos parámetros del análisis de regresión

\begin{tabular}{|c|c|c|c|}
\hline Variable & Estimado & Intervalo de confianza & $\mathrm{p}$ \\
\hline (Intercepto) & $-1.922 \mathrm{e}+03$ & & \\
\hline Población total & $7.653 \mathrm{e}-04$ & $6.1587 \mathrm{e}-04-1.6557 \mathrm{e}-01$ & $* * *$ \\
\hline Temperatura mínima & $-9.810 \mathrm{e}-01$ & $-1.511 e+00--5.204 e-01$ & $* * *$ \\
\hline$\%$ viviendas con piso tierra & $-1.421 e+00$ & $-1.898 e+00--9.543 e-01$ & $* * *$ \\
\hline$\%$ viviendas sin agua entubada & $-7.141 \mathrm{e}-01$ & $-9.7523 e-01--4.6591 e-01$ & $* * *$ \\
\hline $\begin{array}{l}\text { \% Población de } 3 \text { años y más que habla } \\
\text { lengua indígena y no español }\end{array}$ & $-1.802 \mathrm{e}+00$ & $-2.896 e+00--6.845 e-01$ & $* * *$ \\
\hline \% Población de 15 años y más analfabeta & $3.083 \mathrm{e}+00$ & $2.3270 \mathrm{e}+00-3.7993 \mathrm{e}+00$ & $* * *$ \\
\hline \% Población derechohabiente a servicios & $1.902 \mathrm{e}+01$ & $1.3356 \mathrm{e}+01-2.4217 \mathrm{e}+01$ & $* * *$ \\
\hline de salud & & & \\
\hline $\begin{array}{l}\% \text { de viviendas con algún nivel de } \\
\text { hacinamiento }\end{array}$ & $8.758 \mathrm{e}-01$ & $4.5355 \mathrm{e}-01-1.3162 \mathrm{e}+00$ & $* * *$ \\
\hline $\begin{array}{l}\text { \% Viviendas particulares habitadas que } \\
\text { disponen de luz, agua y drenaje }\end{array}$ & $-9.541 \mathrm{e}-01$ & $-1.224 e+00--6.827 e-01$ & $* * *$ \\
\hline
\end{tabular}

El modelo final de regresión no espacial (el modelo con el más bajo valor de AIC), incluía las siguientes covariables: población total, temperatura mínima, porcentaje de ocupantes en viviendas con piso de tierra, porcentaje de ocupantes en viviendas sin agua entubada, población de tres años y más que habla alguna lengua indígena y no habla español, población de 15 años y más analfabeta, 
población derechohabiente a servicios de salud, porcentaje de viviendas con algún nivel de hacinamiento y viviendas que disponen de luz eléctrica, agua entubada de la red pública y drenaje. (Cuadro 2). Ajustando para los efectos de las otras covariables, pero no representando la autocorrelación espacial en los datos, porcentaje de viviendas con piso de tierra, población de tres años y más que habla alguna lengua indígena y no habla español, produce una correlación negativa con el total de casos de enfermos de Chagas, lo que era contrario a la asociación observada durante el análisis univariado. porcentaje de ocupantes en viviendas sin agua entubada también se correlacionó negativamente con el resultado, mientras que el resto de las variables demostraron ser un factor de riesgo. La prueba I de Moran no fue muy clara en la detección de autocorrelación espacial en el modelo de correlación no espacial de Poisson, aunque un tanto dispersa, el patrón puede deberse a la casualidad, lo que indica que las covariables incluidas en el modelo no espacial no explicaban plenamente la variación espacial de la enfermedad de Chagas. Por lo tanto, un análisis de regresión espacial de Poisson fue ajustado utilizando las covariables del modelo de regresión no espacial, para hacer frente a la autocorrelación espacial de los residuales. (Cuadro 2).

Cuadro 3. Parámetros finales estimados del modelo multivariable que incluye efectos fijos y efectos aleatorios correlacionados y no correlacionados

\begin{tabular}{|l|l|l|}
\hline Variable & Media posterior(DS $\left.{ }^{*}\right)$ & Rate Ratio $\left(\mathbf{9 5 \%} \mathbf{C r I}^{\dagger}\right)$ \\
\hline Intercept & $-195.3(12.31)$ & \\
\hline Población total & $-0.05229(0.1132)$ & $0.0053(-0.2409-0.1522)$ \\
\hline Viviendas con piso de tierra & $0.3035(1.087)$ & $0.05141(-1.413-2.02)$ \\
\hline Población de 3 años habla lengua indígena y no español & $0.6079(1.755)$ & $0.0828(-2.029-4.404)$ \\
\hline Población de 15 años y más analfabeta & $-0.8239(2.552)$ & $0.1207(-5.446-3.284)$ \\
\hline Población con derechohabiencia a los servicios de salud & $2.069(0.1664)$ & $0.007857(1.737-2.357)$ \\
\hline Viviendas con hacinamiento & $0.9796(0.2735)$ & $0.01292(0.5446-1.552)$ \\
\hline Viviendas con servicios & $0.1129(0.2464)$ & $0.01163(-0.3756-0.5685)$ \\
\hline Efectos aleatorios no correlacionados & $326.6(893.7)$ & \\
\hline Efectos aleatorios correlacionados & $0.000295(0.000106)$ & \\
\hline *Desviación estándar; 'Intervalo de confianza & & \\
\hline
\end{tabular}

El modelo multivariado que contiene efectos fijos y efectos aleatorios espacialmente correlacionados y no correlacionados, tuvo el más bajo DIC. (Cuadro 3) Los resultados del modelo mostraron correlaciones positivas estadísticamente significativas con la enfermedad de Chagas para viviendas con piso de tierra, población menor de tres años que habla lengua indígena y no español, con derechohabiencia a servicios de salud, viviendas con hacinamiento y servicios. La población total y la población de 15 años y más analfabeta se asociaron negativamente con la enfermedad de Chagas. 
Los mapas de riesgo relativo de acuerdo solo a efectos fijos y a efectos fijos y efectos aleatorios se muestran en las figuras 4 y $\mathbf{5}$, respectivamente. De acuerdo con ambas figuras el mayor riesgo relativo para la enfermedad de Chagas se localiza en los municipios del centro y sur de la Huasteca Potosina, lo que coincide con la ubicación del número de casos del periodo analizado. Sobresale en caso del municipio de El Naranjo, que presenta una alta prevalencia de la enfermedad (Figura 3), pero en el análisis bayesiano no presenta un riesgo alto (Figuras 4 y 5), lo que se debe a que esta área administrativa se encuentra en una zona con características diferentes a la zona media y sur de la Huasteca; por ejemplo, solo $0,7 \%$ de su población es indígena contra $35 \%$ de población indígena de toda el área, en general la parte norte de la Huasteca se caracteriza por tener un grado más alto de industrialización, así como un mayor índice de desarrollo humano y un menor grado de marginación comparado con el resto de la zona, lo que se ve reflejado en un menor número de viviendas con piso de tierra y sin agua entubada, menor analfabetismo, mayor derechohabiencia a servicios de salud entre otras, por lo que debemos tomar con reserva los casos reportados en este sitio, ya que probablemente sea ahí donde se hayan contabilizado, pero tal vez hayan ocurrido en un lugar diferente.

\section{DISCUSIÓN}

Cuantificar el efecto de las variables climáticas, ambientales y socioeconómicas sobre la incidencia de la enfermedad de Chagas permite comprender la epidemiología de la enfermedad y la distribución espacial permitiendo la mejora de las iniciativas de control de enfermedades. El análisis espacial presentado aquí muestra que la incidencia de la enfermedad de Chagas incrementa con las variables material del piso de las viviendas (piso de tierra), el acceso a servicios (viviendas sin agua, luz y drenaje), analfabetismo; un alto riesgo de incidencia de la enfermedad se presenta en áreas con mayor porcentaje de población indígena y sin acceso a servicios de salud (parte centro y sur de la huasteca) y un bajo riesgo se presenta en la zona norte (Figura 5).

Esta enfermedad que se encuentra asociada a la pobreza y las malas condiciones de la vivienda; se localiza ampliamente distribuida, en las áreas rurales de Latinoamérica y en zonas marginadas de las grandes ciudades principalmente (2). La enfermedad de Chagas, más que ninguna otra, está íntimamente ligada con el desarrollo económico y social (3). En este estudio, basados en el análisis de regresión de Poisson univariado, encontramos que la incidencia de la enfermedad de Chagas en la Huasteca Potosina se incrementa con el analfabetismo, la carencia de servicios básicos en las viviendas, el material de las mismas y la falta de servicios de salud, variables que permiten un hábitat 
idóneo para la chinche transmisora, lo que favorece un contacto de ésta con la población; como puede observarse en las Figuras 4 y 5, a diferencia de la zona norte, donde las condiciones socioeconómicas de los habitantes contrastan con los de la zona centro y sur, lo que se ve reflejado en una disminución de casos de enfermos de Chagas.

La importancia del tipo y servicios con que cuentan las viviendas en las que se ha detectado la presencia de la enfermedad indica que los programas de atención y/o control de esta enfermedad deberían ir dirigidos a un cambio en la infraestructura y tipo de construcciones en esta zona, ya que mientras en esas localidades las casas sigan teniendo las condiciones idóneas para la presencia de la chinche de nada servirán los programas dirigidos al control vectorial.

La importancia del porcentaje de población que solamente habla lengua indígena puede deberse a una combinación de factores. En México, este grupo es de los más vulnerables ya que presenta un alto y muy alto grado de marginación (24), viven en zonas de difícil acceso, lo que dificulta la cobertura de servicios de salud e infraestructura de servicios básicos (agua, luz, drenaje, educación) y en caso de la Huasteca Potosina, presenta características ambientales que propician el desarrollo del vector. Por lo tanto, el porcentaje de población indígena puede estar actuando como sustituto de una combinación de estos factores más que como una correlación significativa que se relacione en términos de su propio impacto en presencia de la enfermedad. Los municipios con un mayor ingreso (reflejado en el porcentaje de viviendas que cuentan con servicios básicos) presentan menor riesgo de presentar la enfermedad de Chagas, lo que refuerza una relación con la situación socioeconómica, así como variables climáticas y ambientales.

La población con hogares censales indígenas fue una variable que correlacionó con la incidencia de la enfermedad de Chagas en el análisis univariado (Cuadro 1), pero que perdió significancia en el análisis final. Esto pudo deberse a la incorporación de otras variables socioeconómicas. Por otro lado, la inclusión del porcentaje de población de tres años y más que solamente habla lengua indígena en el análisis multivariado, sugiere que la incidencia más alta no se presenta en lugares donde existen hogares indígenas, sino en sitios donde la población no habla español, los cuales no necesariamente tienen que coincidir.

El análisis de regresión Poisson univariado indica que todas las variables evaluadas, a excepción de $\%$ de viviendas sin drenaje y sin energía eléctrica, población ocupada sin ingresos de hasta dos salarios mínimos y las viviendas particulares habitadas sin ningún bien, fueron significativamente correlacionadas con la incidencia de la enfermedad de Chagas durante el periodo de estudio. La mayor incidencia de la enfermedad se presentó en zonas donde la mayor parte de la población es indígena. 
La forma en que se distribuyen los indígenas a lo largo de la Huasteca no es homogénea, habiendo una menor presencia de ellos al norte de la región, mientras que al centro y sur hay una mayor concentración (25). Los poblados y comunidades en los que se asientan comúnmente carecen de servicios básicos derivado del pequeño tamaño en que se constituyen y de sus ubicaciones, zonas en el interior de las sierras por lo regular segregadas, con casas construidas de materiales como palma y madera, lo que resulta contrastante con las zonas urbanas en las que suelen ubicase principalmente población no indígena, zonas bajas con casas construidas de materiales como el concreto, tabique, etc.

El análisis presentado se realizó usando una inferencia bayesiana. Si bien los métodos bayesianos pueden ser computacionalmente intensivos y requieren altos costos iniciales en términos de formación y comprensión, los resultados que se obtienen ofrecen un marco sólido para el modelado de bases de datos jerárquicos y auto-correlacionadas y permiten la incorporación de la incertidumbre en los parámetros desconocidos del modelo (20). Nuestros resultados deben tomarse con la consideración de las limitaciones de la investigación. Debido a los diferentes tamaños de las áreas administrativas utilizadas en el análisis, los valores utilizados para varias de las covariables (por ejemplo, temperatura mínima, media y máxima, precipitación) tiene diferentes niveles de precisión (es decir, la precipitación dentro de un área administrativa pequeña tendrá una menor varianza que dentro de un área administrativa grande, dependiendo del número de estaciones meteorológicas); estas diferencias pueden tener un impacto en la estimación de los parámetros y niveles de significancia. Además, la referencia espacial de los datos de enfermos de Chagas donde se produjo la infección se basa en la presunta localización donde se produjo, aunque puede ser difícil determinar la ubicación precisa donde fue adquirida. No es posible evaluar la fiabilidad de estos lugares, pero la agregación de casos en áreas administrativas ayudará a negar cualquier imprecisión en la georreferenciación.

Si bien es cierto que está ampliamente documentada la influencia de las variables climáticas en la distribución de vectores de la enfermedad (19, 26-30), en este estudio, dichas variables no resultaron significativas, problablemente porque lo que se está modelando no es la presencia de los vectores, sino la enfermedad, y ésta puede ser transmitida no solamente por la picadura del insecto, sino también por transfusiones de sangre, vía congénita y actualmente algunos brotes recientes se han dado por transmisión oral (4-5,31-32), lo que pudiera explicar por qué en este estudio dichas variables no fueron significativas. 
La aplicación de la regresión de Poisson, incluyendo la regresión espacial y no espacial, ha puesto de relieve la importancia de una serie de factores en la distribución espacial de la enfermedad de Chagas en la Huasteca Potosina. Esta investigación ha aportado evidencias de un mayor riesgo de la enfermedad de Chagas en áreas donde la población es indígena. En la Huasteca Potosina, esta población habita en áreas de difícil acceso, que carecen de servicios básicos, de tamaño pequeño y con casas construidas de materiales como palma y madera. Los programas dirigidos al control vectorial deben tomar en cuenta estas consideraciones, ya que las características de la zona favorecen una infestación estacional de los vectores, es decir, se lleva a cabo un control vectorial, pero al no modificar la infraestructura de las localidades, estas siguen permitiendo una reinfestación posterior.

\section{Referencias}

1. WHO Enfermedad de Chagas: control y eliminación. 63.a Asamblea Mundial de la Salud. [en línea] WHO; 2010. $\begin{array}{lllll}\text { Fecha de } & \text { acceso } & \text { Enero } & \text { Disponible } & \text { en: }\end{array}$ http://www.paho.org/hq/index.php?option=com_docman\&task=doc_view\&gid=15376\&Itemid=

2. Coura JR, Borges-Pereira J. Chagas disease: 100 years after its discovery. A systemic review. Acta trop. 2010 Jul; 115 (1-2): 5-13.

3. Secretaría de Salud. Programa de acción específico 2007-2012. Otras enfermedades transmitidas por vector. Subsecretaría de Prevención y Promoción de la Salud. [En línea] SSA; 2012; Fecha de acceso Septiembre 2015. Disponible http://www.cdi.salud.gob.mx:8080/BasesCDI/Archivos/Enfermedadestransmitidasporvector/etv.pdf

4. WHO Control of Chagas'disease. Report of a WHO Expert Committee. [En línea] WHO; 1991. Fecha de acceso Noviembre 2015. Disponible en: http://www.ircwash.org/sites/default/files/WHO-1991-Control.pdf

5. WHO Control of Chagas'disease. Second Report of the WHO Expert Committee. [En línea] WHO; 2002. Fecha de acceso Noviembre 2015. Disponible en: http://apps.who.int/iris/bitstream/10665/42443/1/WHO_TRS_905.pdf

6. Abad-Franch F, Santos WS, Schofield CJ. Research needs for Chagas disease prevention. Acta trop. 2010 Jul; 115 (1-2): 44-54.

7. Wendel S. Transfusion transmitted Chagas disease: is it really under control?. Acta trop. 2010 Jul; 115 (1-2): 28 34.

8. Zeledón R, Montenegro VM, Zeledón O. Evidence of colonization of man-made ecotopes by Triatoma dimidiata (Latreille, 1811) in Costa Rica. Mem Inst Oswaldo Cruz. 2001 Jul; 96 (5): 659-60.

9. Tabaru Y, Monroy C, Rodas A, Mejia M, Rosales R. The geographical distribution of vectors of Chagas disease and populations at risk of infection in Guatemala. Med. Entomol. Zool. 1999 Oct; 50(1): 9-17.

10. Sasaki H, Rosales R, Tabaru Y. Host feeding profiles of Rhodnius prolixus and Triatoma dimidiata in Guatemala (Hemiptera: Reduviidae: Triatominae). Med. Entomol. Zool. 2003 Jun; 54 (3): 283-89.

11. Petana WB. American trypanosomiasis in British honduras. X. Natural habitats and ecology of Triatoma dimidiata (Hemiptera, Reduviidae) in the El Cayo and Toledo districts, and the prevalence of infection with Trypanosoma (Schizotrypanum) cruzi in the wild-caught bugs. Ann Trop Med Parasitol. 1971 Jun; 65(2): 169-78.

12. Zeledón R, Rabinovich JE. Chagas disease: an ecological appraisal with special emphasis on its insect vectors. Annu Rev Entom. 1981 Ene; 26: 101-33.

13. Monroy MC, Bustamante DM, Rodas AG, Enriquez ME, Rosales RG. Habitats, dispersion and invasion of sylvatic Triatoma dimidiata (Hemiptera: Reduviidae: Triatominae) in Peten, Guatemala. J Med Entomol. 2003 Nov; 40(6): 800-6.

14. Arzube M. Investigación de la fuente alimenticia de Triatoma dimidiata Latreille 1811 (Hemiptera: Reduviidae) mediante la reacción de precipitina. Rev Ecuatoriana de Higiene Med Trop. 1966 May; 23, 137-52.

15. Zeledón R, Solano G, Zúñiga A, Swartzwelder JC. Biology and ethology of Triatoma dimidiata (Latreille, 1811). III. Habitat and blood sources. J Med Entomol. 1973 Jul; 10(4): 363-70. 
16. Rosabal R. Hallazgo de Triatoma dimidiata silvestre infectado por Schizotrypanum cruzi en Guapiles, provincia de Limón, Costa Rica. Arch Col Med El Salvador. 1969 Mar; 22(1): 77-78.

17. Schofield CJ, Organización Panamericana de la Salud. Evolución y control del Triatoma dimidiata. Taller para el establecimiento de pautas técnicas en el control de Triatoma dimidiata. 2002 Mar; 12-8.

18. Cruz-Reyes A, Pickering-López JM. Chagas disease in Mexico: an analysis of geographical distribution during the past 76 years-A review. Mem Inst Oswaldo Cruz. 2006 Jun; 101(4):345-54.

19. Dumonteil E, Gourbiere S. Predicting Triatoma dimidiata abundance and infection rate: a risk map for natural transmission of Chagas disease in the Yucatan peninsula of Mexico. Am J Trop Med Hyg. 2004 May; 70(5): 5149.

20. Best N, Richardson S, Thomson A. A comparison of Bayesian spatial models for disease mapping. Stats Methods Med Res. 2005 Feb; 14(1): 35-59.

21. Lawson AB. Bayesian disease mapping: hierarchical modeling in spatial epidemiology. 2nd ed. Boca Raton: CRC Press; 2013

22. Instituto Nacional de Estadística y Geografía. Censo de población y vivienda, 2010. Principales resultados por localidad (ITER) [Fecha de acceso: 10 de septiembre del 2015] Disponible en: http://www.inegi.org.mx/sistemas/consulta_resultados/iter2010.aspx?c=27329\&s=est

23. ESRI. ArcGIS Desktop: Release Redlands CA: Environmental Systems Research Institute. 2011

24. Kéry M. Introduction to WinBUGS for ecologists: Bayesian approach to regression, ANOVA, mixed models and related analyses. Burlington: Academic Press; 2010.

25. Gallardo-Arias P. Huastecos de San Luis Potosí: pueblos indígenas del México contemporáneo. CDI PNUD. 2004

26. Gorla DE. Variables ambientales registradas por sensores remotos como indicadores de la distribución geográfica de Triatoma infestans. Ecología Austral. 2002 Dic; 12:117-27.

27. Rodrigueiro M, Grola D. Latitudinal gradient in species richness of the New World Triatominae (Reduviidae). Global Ecol. Biogeograph. 2004 Ene; 13:75-84

28. Bustamante DM, Monry C, Rodas A, Juarez J, Malone J. Environmental determinants of the distribution of Chagas vectors in south-eastern Guatemala. Geospatial Healt. 2008 May; 2:199-211

29. Arboleda S, Gorla D, Porcasi X, Saldaña A, Calzada J, Jaramillo N. Development of a geographical distribution model of Rhodnius pallescens Barber, 1932 using environmental data recorded by remote sensing. Infect Genet Evol. 2009 Jul; 9: 441-8.

30. Carbajal de la Fuente AL, Porcasi X, Noireau F, Diotaiuti L, Gorla D. The association between geographic distribution of Triatoma Pseudomaculata and Triatoma Wygodzinsky (Hemiptera: Reduviidae) with environmental variables recorded by remote sensors. Infec Genet Evol. 2009 Ene; 9:54-61.

31. Toso A, Vial F, Galanti N. Transmisión de la enfermedad de Chagas por vía oral. Rev Med Chile. 2011 Feb; 139:258-266.

32. Shikanai-Yasuda MA, Carvalho NB. Oral transmission of Chagas Disease. Clin Infect Dis. 2012 Ene; 845-52. 
Medina-Garza et al

\section{Imágenes}

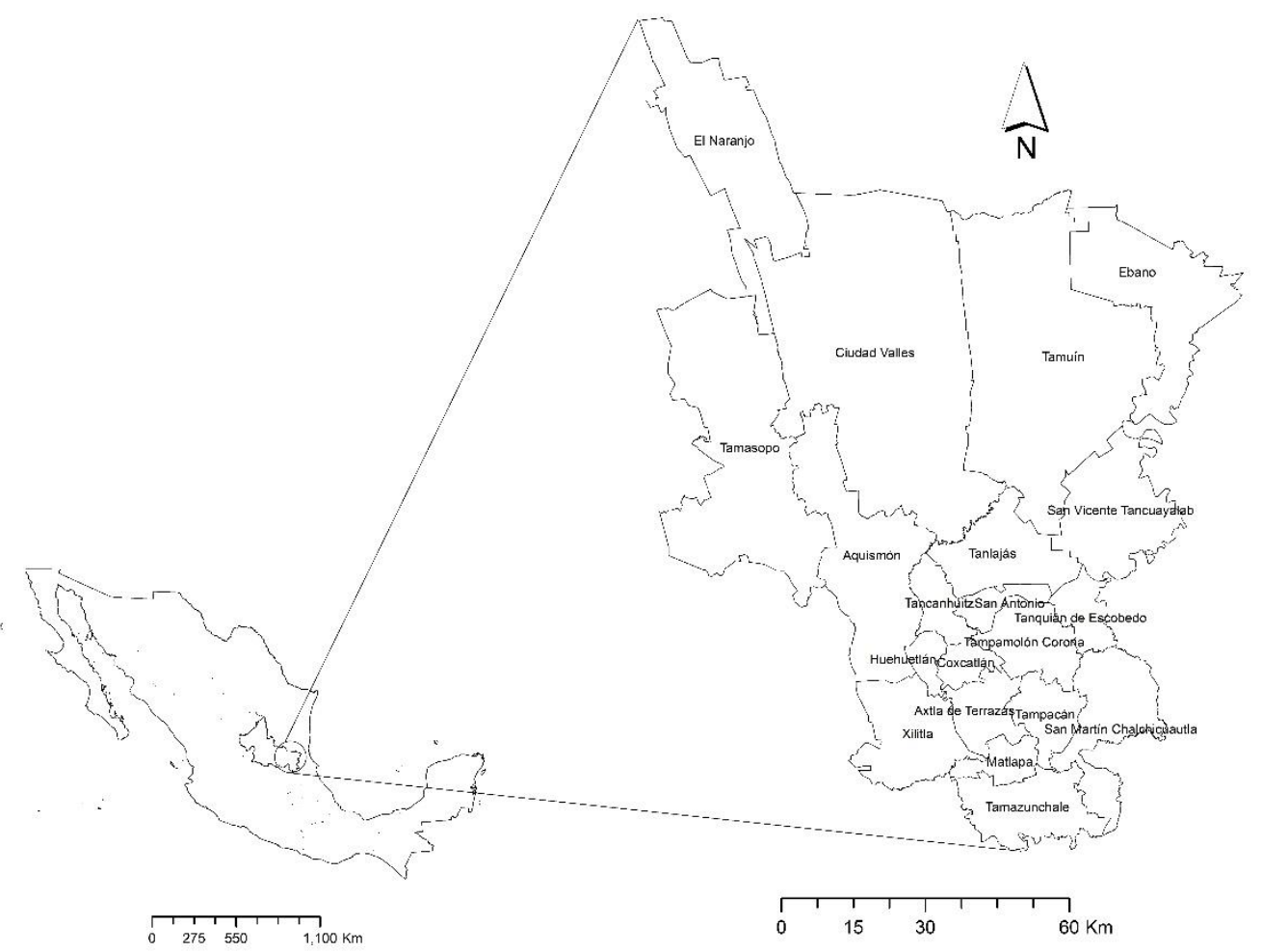

Figura 1. Ubicación de la Huasteca Potosina. Esta zona abarca 20 municipios del Estado de San Luis Potosí. 


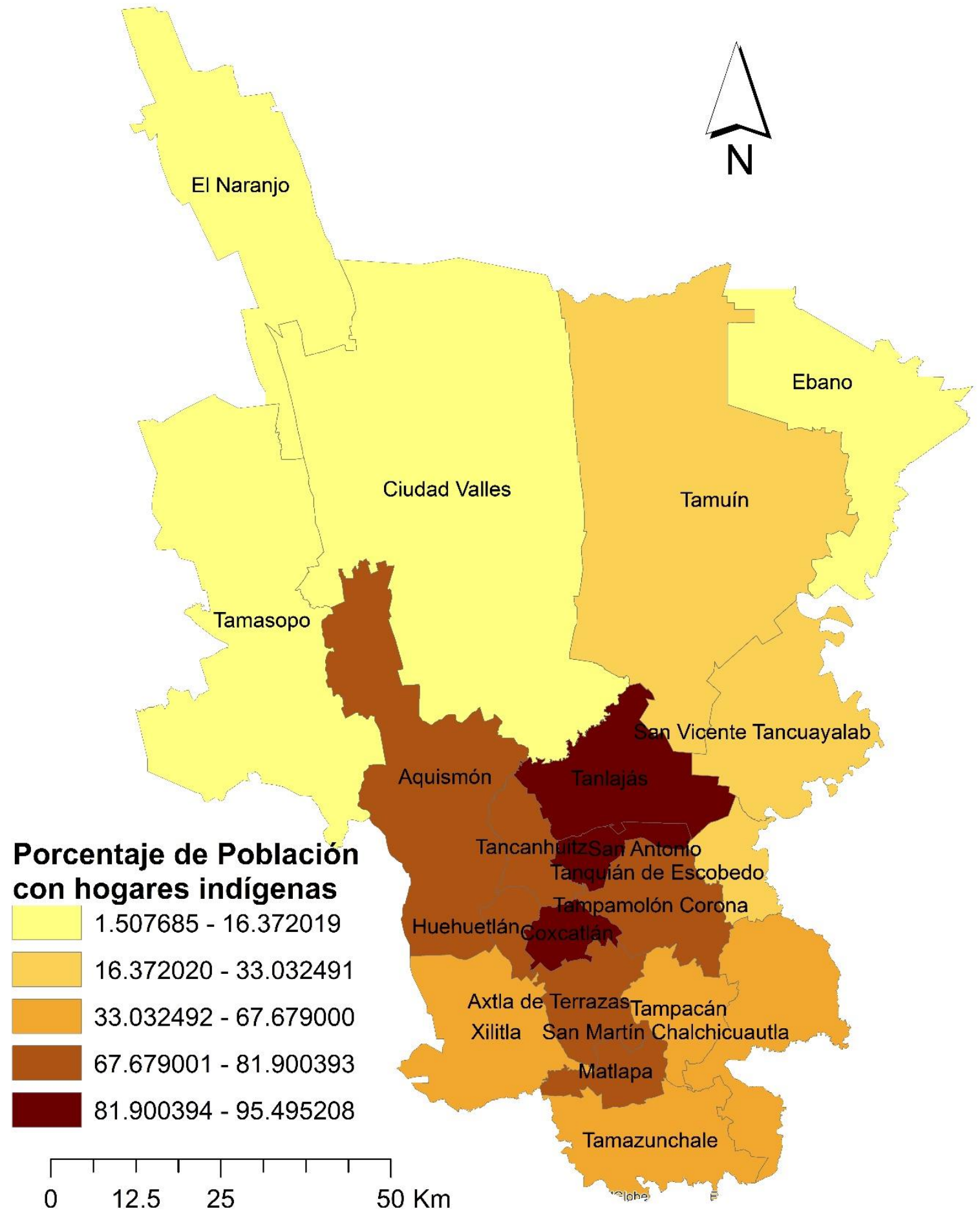

Figura 2. Total, de personas que forman hogares censales donde el jefe del hogar o su cónyuge hablan alguna lengua indígena. Los municipios del centro y sur de la Huasteca son en los que en la mayoría de los hogares habitan personas que hablan alguna lengua indígena. 
Medina-Garza et al

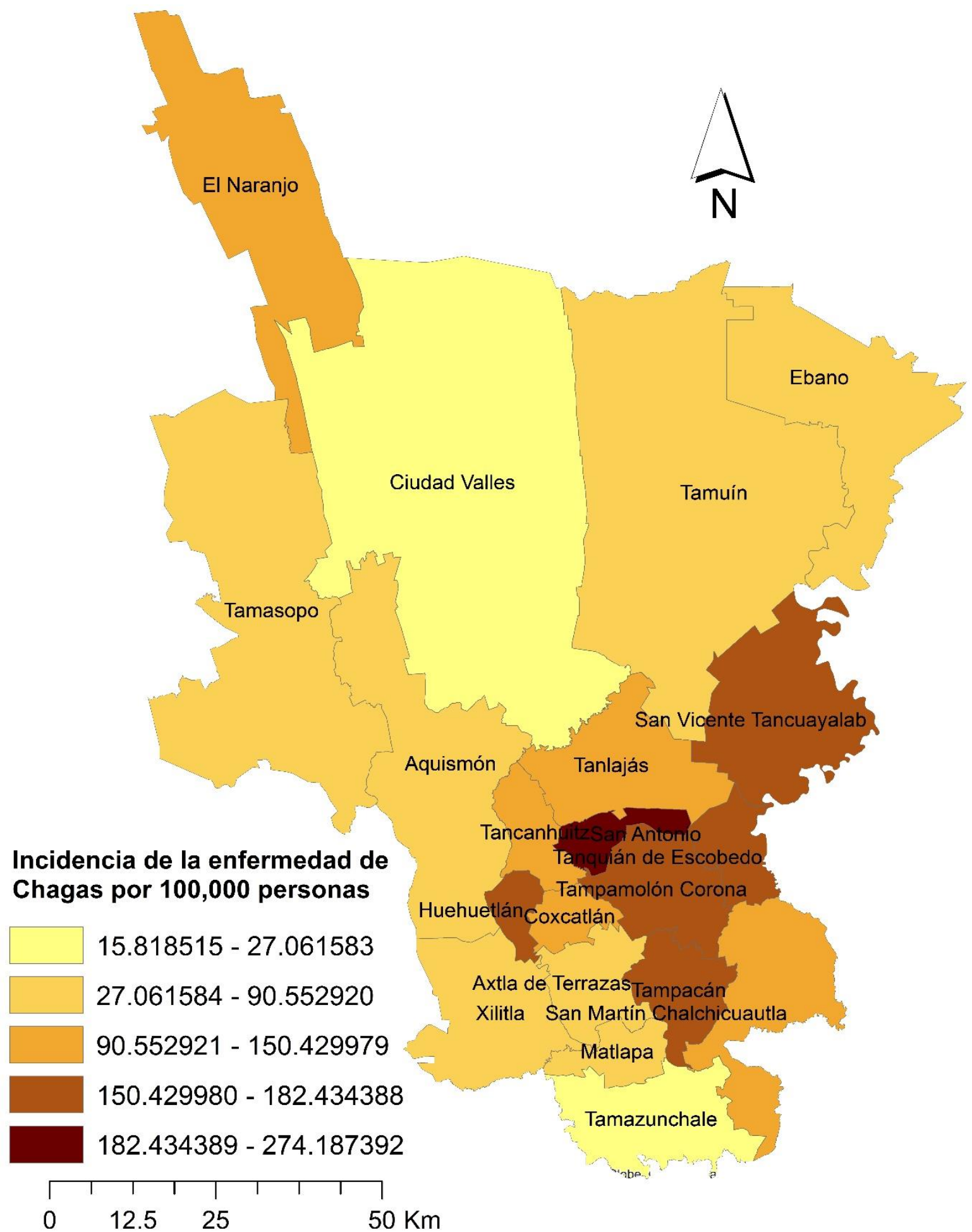

Figura 3. Prevalencia por 100000 personas de enfermos de Chagas por municipio en la Huasteca Potosina. 2006 - 2012. La mayor prevalencia de la enfermedad se da en los municipios del centro y sur de la Huasteca, a excepción de El Naranjo, ubicado en la Huasteca norte 


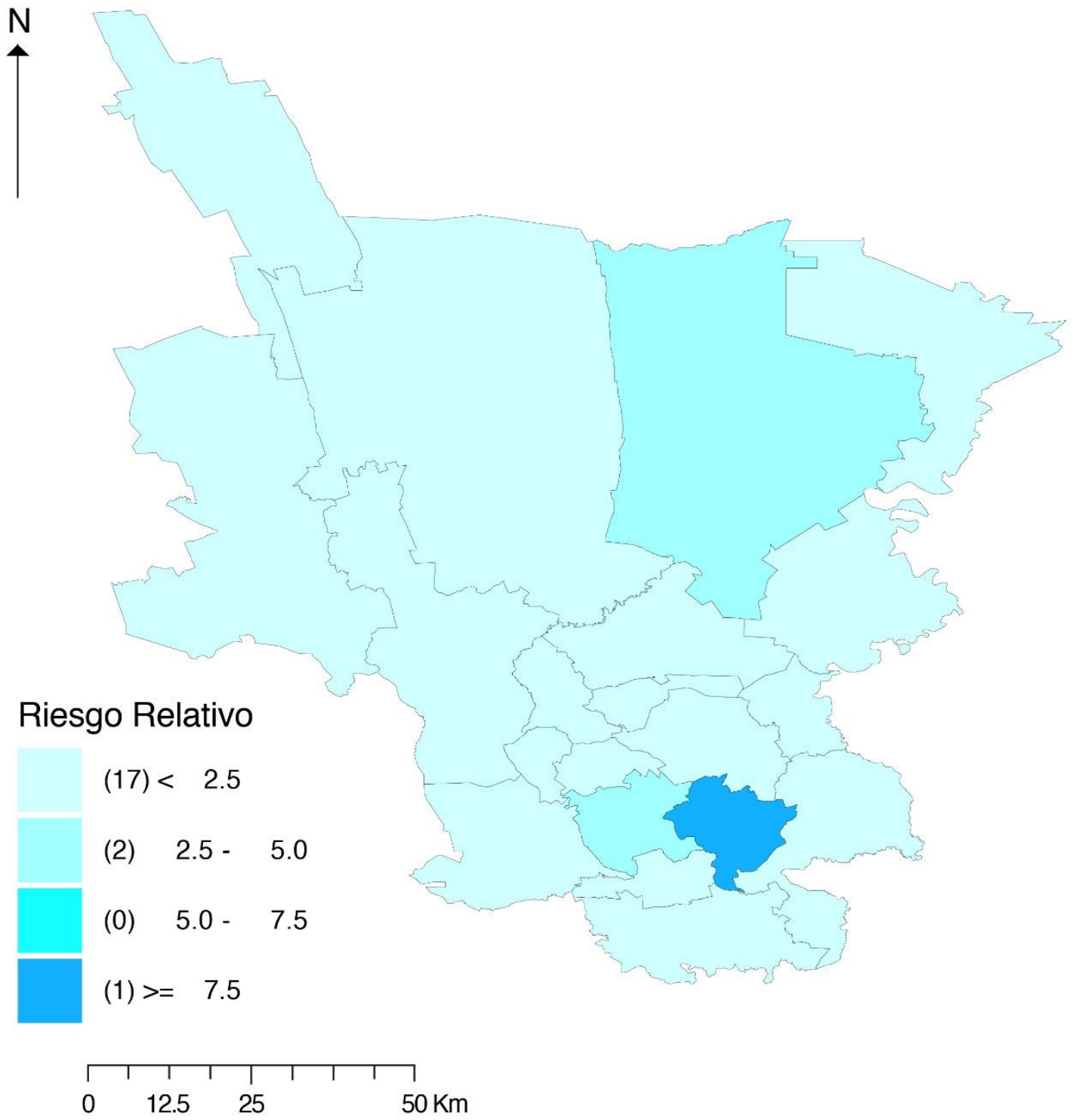

Figura 4. Riesgo relativo de la enfermedad de Chagas en municipios de la Huasteca Potosina, de acuerdo solo a efectos fijos. 
Medina-Garza et al

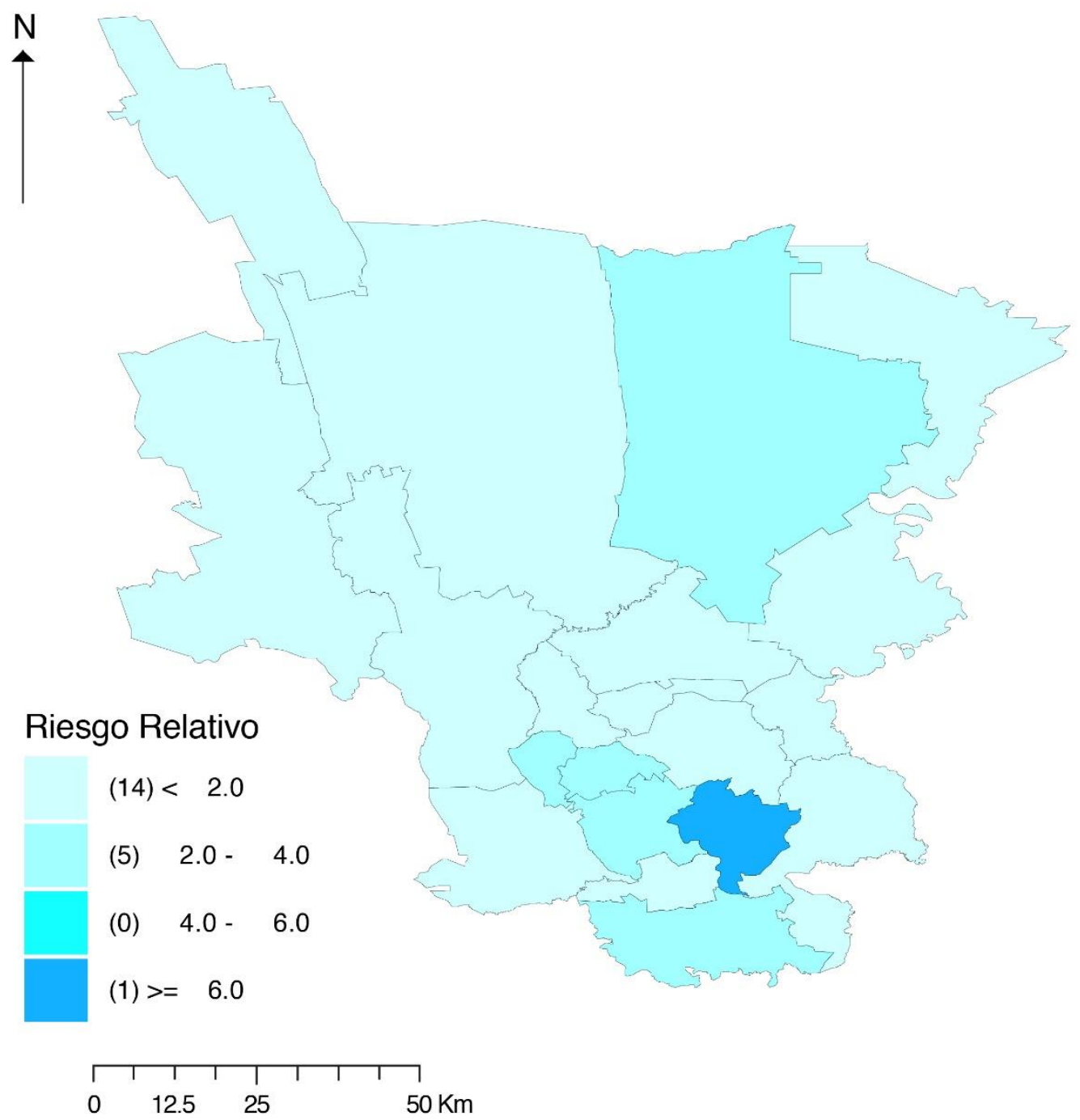

Figura 5. Riesgo relativo de la enfermedad de Chagas en municipios de la Huasteca Potosina, de acuerdo a efectos fijos y efectos aleatorios. 